\title{
THE DEPTHS OF HYDROGEN AND HELIUM BUBBLES IN TUNGSTEN: A COMPARISON
}

\author{
K. O. E. HENRIKSSON, ${ }^{*}$ K. NORDLUND, A. KRASHENINNIKOV, and J. KEINONEN \\ University of Helsinki, Accelerator Laboratory, P.O. Box 43, FI-00014 Finland
}

Received September 14, 2005

Accepted for Publication January 16, 2006

The role of self-trapping and defect trapping of hydrogen and helium implanted into tungsten has been investigated using density functional theory (DFT) calculations, molecular dynamics simulations, and kinetic Monte Carlo simulations (KMCSs). The potential energy curves of hydrogen or helium pairs were obtained by molecular dynamics, and the energy of the most essential states was checked with DFT. Under assumptions of bubble formation due to trapping by similiar impurity atoms (self-trapping) or defects, KMCSs were carried out using parameters from implantation experiments. The results indicate that self-trapping plays no (or a very small) role in hydrogen bubble formation, whereas helium bubbles form due to strong self-trapping.

KEYWORDS: light gases, bubbles, atomistic simulations

\section{INTRODUCTION}

It is known that light gases such as hydrogen and helium implanted into solids can form gas bubbles in the target. ${ }^{1}$ Under appropriate conditions these bubbles may grow into larger bubbles, becoming visible on the target surface as small bumps, also known as blisters. Under prolonged implantation these blisters may rupture, ejecting gas atoms and fragments of the solid. Blistering could be harmful in, e.g., fusion reactors, especially when the plasma-facing materials contain heavy chemical elements, e.g., tungsten, since energy loss in the plasma is proportional to the proton number ( $Z$ value) of the atoms contaminating the plasma. ${ }^{2}$

In 1858 Plücker observed that repeated discharges in various gas atmospheres degraded over time (Ref. 1 and references therein). His explanation was that gas atoms were lost to the electrodes, in effect implanted, into the cathode in the case of positive ions. The first detailed

\footnotetext{
*E-mail: krister.henriksson@helsinki.fi
}

blistering study (according to Ref. 1) after Plücker's initial discovery was carried out by Primak, Dayal, and Edwards ${ }^{3}$ in 1963, using 100- to 450-keV hydrogen and helium irradiation of silicon. In the same year Primak ${ }^{4}$ also observed blistering and pitting (the formation of holes in the surface) on various insulators (corundum, spinel, rutile, and peridot), caused by $100-$ to $140-\mathrm{keV}$ hydrogen and helium irradiation.

\section{I.A. Hydrogen and Helium Bubbles in Tungsten}

Turning to elastically hard metals, and focusing on ion energies of $10 \mathrm{keV}$ or lower, it should be noted that blisters and the first stage in blister production, the formation of bubbles, have been observed for hydrogen (or deuterium $)^{5-16}$ and helium ${ }^{10-12,16-26}$ implanted into singlecrystalline and polycrystalline tungsten under different experimental conditions. The essential difference between hydrogen and helium bubbles in tungsten is in the depth of the bubbles: Hydrogen bubbles tend to form at depths that are several orders of magnitude times the projected range, whereas helium bubbles often are located at depths close to the projected range, especially for low temperatures, say $T \approx 300 \mathrm{~K}$. For example, the helium bubble depths-measured using field ion microscopy (FIM) - in Ref. 18, with $T=300 \mathrm{~K}$, are very close to projected range values calculated using the stopping and range of ions in matter (SRIM) program. ${ }^{27}$

On the other hand, implantation of $110 \mathrm{eV}$ at $850 \mathrm{~K}$ (Ref. 6), $500 \mathrm{eV}$ at $500 \mathrm{~K}$ (Ref. 8), and $1 \mathrm{keV}$ at $300 \mathrm{~K}$ (Ref. 10) deuterium produced bubbles at depths of 5 and $10 \mu \mathrm{m}$ (Ref. 6) and blisters with lid thicknesses of $10 \mu \mathrm{m}$ (Ref. 8) and $0.5 \mu \mathrm{m}$ (Ref. 10). The projected ranges of hydrogen at these energies are $\sim 24,62$, and $97 \AA$, respectively, and are possibly slightly larger for deuterium. Nevertheless, the experimental blister depths deviate by several orders of magnitude from the projected ranges. It is unclear what causes this large discrepancy.

\section{I.B. Differences in the Diffusivities}

In general, similar temperatures have been used for hydrogen (or deuterium) and helium implantation, but 
the fluxes and fluences have in most cases been larger for hydrogen implantation than for helium. If the time between insertion of ions is shorter for hydrogen than for helium, then hydrogen should be less able to escape the implantation region and therefore should cluster more closely to the surface than helium, all other conditions being similar. The fluence should mainly affect the size of the bubbles, not their depth, at least in the beginning of the bubble formation process. As bubbles grow, they will obstruct the inflow of ions, thereby influencing the subsequent average depth of both atoms and bubbles.

It is possible that different migration rates could explain the different bubble depths, if hydrogen diffuses significantly faster than helium at similar temperatures. There exist several different values for the diffusivity $D(T)=D_{0} e^{-E_{M} /\left(k_{B} T\right)}$ of hydrogen and its isotopes in tungsten, measured over various temperature ranges. ${ }^{28-33}$ The result that is recommended by at least Causey and Venhaus $^{2}$ and Causey ${ }^{34}$ is that of Frauenfelder: $\left(D_{0}, E_{M}\right)=$ $\left(4.1_{-2.0}^{+5.0} \times 10^{-7} \mathrm{~m}^{2} \mathrm{~s}^{-1}, 0.39 \pm 0.09 \mathrm{eV}\right)$ (Ref. 30), obtained for temperatures of 1100 to $2400 \mathrm{~K}$. For helium we have from the literature $\left(D_{0}, E_{M}\right)=\left(4.7_{-3.3}^{+9.2} \times\right.$ $10^{-7} \mathrm{~m}^{2} \mathrm{~s}^{-1}, 0.28 \mathrm{eV}$ ) (Ref. 35). The ratio of the diffusivities is $D_{\mathrm{H}} / D_{\mathrm{He}}=(0.9 \pm 1.3) \exp [-(0.11 \pm 0.09) /$ $\left.\left(k_{B} T\right)\right]$. At $T=300 \mathrm{~K}$, the ratio is $0.01 \pm 0.05$, i.e., quite small, which indicates that helium migrates much faster than hydrogen.

However, the migration of hydrogen in tungsten is complicated by the possibility of nonclassical (quantummechanical) diffusion at "low" temperatures, especially if "low" is close to $300 \mathrm{~K}$. At least one study, by Macrander and Seidman, ${ }^{36}$ indicates that hydrogen is able to diffuse quantum-mechanically in tungsten. In this study, $200-\mathrm{eV}$ hydrogen molecules were implanted into singlecrystalline regions of a tungsten sample held at $29 \mathrm{~K}$. The sample was then analyzed by FIM. The analysis showed that the hydrogen atoms had left the implanted region. From the data, Macrander and Seidman estimated a lower bound of the diffusion constant of $D(29 \mathrm{~K})=(1-10) \times$ $10^{-22} \mathrm{~m}^{2} \mathrm{~s}^{-1}$. In comparison, the Frauenfelder result implies a classical diffusion constant of $6.9 \times 10^{-75} \mathrm{~m}^{2} \mathrm{~s}^{-1}$ at this temperature, i.e., that hydrogen atoms are for all practical purposes immobile.

It is, however, unknown below which threshold temperature quantum-mechanical migration (QMM) of hydrogen and its isotopes becomes important. One may perhaps estimate the threshold temperature in tungsten by investigating other body-centered-cubic (BCC) metals where QMM has been established. From the data for vanadium, niobium, and tantalum in a review of hydrogen diffusion in metals by Wipf ${ }^{37}$ one can calculate a threshold homologous temperature $T_{O Q M} / T_{m}$. Here, $T_{m}$ is the melting temperature in units of kelvin. Using the plots provided by Wipf and appropriate melting temperatures, ${ }^{38}$ one obtains $0.17 T_{m}, 0.10 T_{m}$, and $0.07 T_{m}$ for vanadium, niobium, and tantalum, respectively. Since tantalum and tungsten are adjacent BCC metals (with similar lattice parameters) on the same row in the periodic table, one may perhaps expect that QMM in tungsten becomes important at a threshold homologous temperature similar to that of tantalum. If this can be argued, then a threshold temperature in the neighborhood of $\sim 0.1 T_{m}=370 \mathrm{~K}$, or $\approx 100^{\circ} \mathrm{C}$, is obtained [with the melting temperature $3695 \mathrm{~K}$ (Ref. 38)]. However, the data in Ref. 37 show also that even at a temperature of some $50 \%$ below the onset of QMM, the migration is still at most about one order of magnitude faster than one could expect from extrapolation downward of the classical high-temperature migration curve. Moreover, QMM is observed only for hydrogen and not deuterium or tritium for these metals. ${ }^{37}$ Considering this, we can conclude that even accounting for possible QMM, almost certainly hydrogen and definitely deuterium and tritium do not diffuse faster than helium close to room temperature.

\section{I.C. Trapping of Hydrogen and Helium in Tungsten}

Since hydrogen migrates more slowly or about as fast as helium at room temperature, a difference in migration rates cannot explain the much larger depths of hydrogen bubbles. There are at least two other possibilities for explaining the different depths: (a) the lateralstress model for blistering and (b) different self-trapping or defect-trapping behavior.

In the lateral-stress model of blistering, ${ }^{39,40}$ the formation of blisters is attributed to the buildup of stress, caused by the implanted ions and the irradiation-created damage. The stress has a maximum value at a depth larger than the projected range of the ions. This model for blister formation has not been established firmly, which is also true for the alternative gas-pressure model for blistering. In this latter model the formation of large surface bubbles is thought to be the result of many smaller bubbles in the implantation region cracking into a common volume due to their high pressure. Martynen$\mathrm{ko}^{41}$ has made a comparison of the two models in the case of blistering by helium ions. The conclusion from the calculations, using a simplified interatomic potential for helium, and considering the interplay between helium atoms and vacancies, is that the lateral-stress model would dominate at "low" temperatures (presumably $T / T_{m} \lesssim 0.2$; see Ref. 40, where $T_{m}$ is the melting temperature of the material), and the gas-pressure model at high temperatures. The transition could possibly be identifiable by the change of the surface topography: Blisters with well-defined thicknesses and diameters (their interdependence is predicted by the lateral-stress model) are replaced by exfoliation of flakes and lumps. The condition $T / T_{m} \sim 0.2$ corresponds to $T \sim 740 \mathrm{~K}$ for tungsten. Corresponding conditions for hydrogen are not known to us. In this study we will not try to establish which model, the lateral-stress or the gas-pressure, has more merits. Instead, we can say that provided hydrogen or helium gas bubbles play an important role in 
blister formation, our results should be of relevance to blistering.

There is still one important possibility for the vastly different experimental depths of hydrogen and helium bubbles, and that is different self-trapping and defecttrapping behavior. Picraux ${ }^{42}$ considered the following six defect types that could possibly trap hydrogen atoms: other hydrogen atoms, single vacancies, impurities, host interstitial atoms, extended defects like dislocations (and grain boundaries), and small vacancy or interstitial clusters.

The possibility of self-trapping of hydrogen has previously been discussed in the literature. According to Switendick ${ }^{43}$ there are two highly simplified models for treating hydrogen in metals. These are the proton model and the anion model. In the proton model the electrons from the inserted hydrogen atoms are added at the Fermi level and thus transferred to the "electronic sea" of the solid. In the anion model the hydrogen atoms induce low-lying electronic states, attracting charge to the local neighborhood. In both models, the hydrogen atom is turned into a localized charge. By these general arguments the interaction between two hydrogen atoms close to one another would always be repulsive. Calculations of electron structure by Nørskov ${ }^{44}$ using the jellium model indicate that $\mathrm{H}-\mathrm{H}$ interaction would indeed be repulsive in most metals. According to Nørskov, this is understandable from the $\mathrm{H}_{2}$ induced density of states, which indicate that the antibonding $\mathrm{H}_{2}$ orbital is filled at densities normal for metals. In addition, McLellan ${ }^{45}$ notes there are indications that the $\mathrm{H}-\mathrm{H}$ interaction in vanadium, niobium, and tantalum is very weak, but it is possible that the interaction can change from attractive to repulsive when the hydrogen concentration is increased.

In general, effective medium calculations in combination with experimental findings indicate there is a simple recipe for determining what kinds of defects are able to trap hydrogen. ${ }^{46-48}$ For the high-density metals, anything that locally lowers the electron density will enhance the stability of hydrogen impurities. The list of defects that may trap hydrogen then includes vacancies, voids, and substitutional impurity atoms (which are smaller than the host lattice atoms). Dislocations and interstitials may also provide trapping sites, due to the relaxation of the surrounding lattice. One may order the defects according to how strongly they trap hydrogen in the following way: dislocation, vacancy, and void (or cavity). Here, dislocations provide the weakest trapping, and voids provide the strongest. In addition, since the interstitial electron density in metals is larger than what is optimal for hydrogen, interstitial hydrogen atoms will induce a local expansive relaxation of the lattice. ${ }^{48}$ This in turn may provide nearby locations with weaker-than-normal electron density, at which additional hydrogen atoms may become trapped. In the following we will not be concerned with the exact nature of the defect traps. For our purposes there are only two categories of defect traps: (a) traps that can accommodate only a few impurity atoms (hydrogen, helium) and therefore are unable to grow into bubbles and (b) traps that can grow. For more detailed discussion of various hydrogen traps in tungsten, we refer to a review by Causey. ${ }^{34}$

In the case of helium, there is proof that self-trapping is important for bubble formation. Evidence for helium bubbles has been reported in experiments using nondamaging (or nearly nondamaging) helium irradiation, in both single-crystalline tungsten targets ${ }^{17,18,20,24}$ and polycrystalline ones. ${ }^{12,16,21,23-26}$ Helium bubbles have also been seen in molecular dynamics simulation (MDS) studies. ${ }^{49,50}$ In the computational investigations it was found that helium ions very easily trap each other, forming small clusters. These grow into larger ones by pushing out self-interstitial tungsten atoms, thereby causing interstital crowdions and the punching out of (111) loops.

Damaging $(8-\mathrm{keV})$ hydrogen irradiation of singlecrystalline tungsten samples gives rise to bubbles, as noted by Sakamoto et al. ${ }^{5}$ For nondamaging irradiation there seems to exist a temperature limit for blistering in singlecrystalline tungsten. Tokunaga et al. ${ }^{51}$ have found blisters on single-crystalline (100) tungsten irradiated with $100 \mathrm{eV}$ deuterium using a flux of $10^{22}$ ions $\mathrm{m}^{-2} \mathrm{~s}^{-1}$, up to a fluence of $10^{26} \mathrm{~m}^{-2}$, at a temperature of $343 \mathrm{~K}$, but not at 383,623 , and $1123 \mathrm{~K}$. The diameters of the blisters were between 1 and $2 \mu \mathrm{m}$. On the other hand, Guseva et al. ${ }^{52}$ have reported that no surface damage occurred for $150-\mathrm{eV}$ deuterium irradiation of single-crystalline (111) tungsten using a flux of $7.7 \times 10^{21}$ ions $\mathrm{m}^{-2} \mathrm{~s}^{-1}$, up to a fluence of $10^{25}$ ions $\mathrm{m}^{-2}$, at $770 \mathrm{~K}$. In this latter study scanning electron microscopy was used to visually inspect the surface, and details down to $0.5 \mu \mathrm{m}$ in linear size were clearly visible.

For polycrystalline tungsten samples, reports of blister formation under nondamaging hydrogen (or deuterium) irradiation are far more abundant. ${ }^{6,8-12,14-16,51}$ The sample temperature in these studies ranged from $300 \mathrm{~K}$ (Ref. 10) to $920 \mathrm{~K}$ (Ref. 12). The findings indicate that hydrogen (or deuterium) ions are easily trapped by native defects, such as grain boundaries and those created by the sample manufacturing process (e.g., the layered structure resulting from the roll-press procedure when using powder-metallurgy methods to make tungsten foils ${ }^{14}$ ), but it is not known whether or not self-trapping plays a role.

\section{I.D. Aim of This Work}

In an earlier study ${ }^{53}$ we reported on the binding energies of small hydrogen and helium clusters in tungsten. In this work we provide more details of these calculations, which included density functional theory (DFT) calculations (DFTCs) and MDSs. We also give more results for the depths of self-trapped and defect-trapped bubbles, as determined by kinetic (object) Monte Carlo (KMC) simulations (KMCSs) of experimental setups. 
The central results in the previous work are incorporated into this study. The main goal of the calculations is still the verification of the proposition that self-trapping plays no or a very small role for hydrogen bubble (or blister) formation, whereas helium bubbles form due to strong self-trapping. The experimental observation that hydrogen blisters are formed on single-crystalline tungsten surfaces after nondamaging irradiation below a certain temperature ${ }^{51}$ will be considered in the discussion of our results. These results will not be modeled with KMCSs since the depths of the blisters are not reported.

\section{METHODS}

\section{II.A. MDS Energy Calculations}

Molecular dynamics simulations were carried out in order to obtain the formation energies of helium and hydrogen atoms as well as helium and hydrogen pairs, located at interstitial sites in tungsten. Systems containing 27648 tungsten and two helium or hydrogen atoms were relaxed at zero kelvin and zero pressure using periodic boundary conditions.

Three potentials were employed for the atomic interaction. To describe the W-W interaction when doing calculations relevant for helium in tungsten, a potential based on the Embedded Atom Method by Finnis and Sinclair ${ }^{54}$ was used. Pair potentials were employed for calculations involving He-He (calculated using the DMol package ${ }^{\mathrm{a}}$ ) and He-W (Ref. 49) interactions. At small interatomic distances the potentials were smoothly joined to the universal repulsive Ziegler-Biersack-Littmark potential ${ }^{55}$ to realistically describe strong collisions. The cutoff radius for all the potentials was $4.4 \AA$. The MDS concerning hydrogen in tungsten used new W-W, H-W, and $\mathrm{H}-\mathrm{H}$ potentials developed at our laboratory..$^{5}{ }^{\mathrm{b}}$

\section{II.B. Density Functional Theory Calculations}

The DFTCs of hydrogen and helium energetics in tungsten were performed using the plane wave basis CASTEP code, ${ }^{57}$ implementing the generalized gradient approximation of Perdew et al. ${ }^{58}$ We used ultrasoft pseudopotentials ${ }^{59,60}$ to describe the core electrons. A kinetic energy cutoff of $500 \mathrm{eV}$ was found to converge the en-

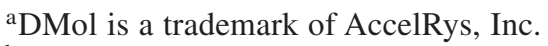

${ }^{b}$ The parameters used for the W-H system are as follows: $\mathrm{H}-\mathrm{H}$, $\gamma=12.33, \mathrm{~S}=2.3432, \beta=1.9436 \AA^{-1}, D_{0}=4.7509 \mathrm{eV}, r_{0}=$ $0.74144 \AA, c=0.0, d=1.0, h=1.0,2 \mu=4.0 \AA^{-1}, R_{\text {cut }}=$ $1.4 \AA ; D=0.3 \AA$ A $\mathrm{W}-\mathrm{W}: \gamma=0.00188227, \mathrm{~S}=1.92708235$, $\beta=1.38527632 \AA^{-1}, D_{0}=5.41860794 \mathrm{eV}, r_{0}=2.34095309 \AA$, $c=2.14968918, d=0.17125622, h=-0.27780144,2 \mu=$ $0.45876488 \AA^{-1}, R_{\text {cut }}=3.5 \AA, D=0.3 \AA$; and W-H: $\gamma=$ $0.0054, \mathrm{~S}=1.2489, \beta=1.52328 \AA^{-1}, D_{0}=2.748 \mathrm{eV}$, $r_{0}=1.727 \AA, c=1.788, d=0.8255, h=0.38912395,2 \mu=$ $0.0 \AA^{-1}, R_{\text {cut }}=2.15 \AA, D=0.2 \AA$ (Ref. 56).
}

ergy difference between the studied configurations to within $0.02 \mathrm{eV}$. The same accuracy was achieved with respect to the $k$-point sampling of the Brillouin zone. In practice, we used $4 k$-points, as control runs with 14 points changed our results by $<0.02 \mathrm{eV}$.

Slabs with the geometry optimized with the MDSs were used as input for the DFTCs. The pure system consisted of 54 tungsten atoms $(3 \times 3 \times 3$ unit cells $)$. First, the geometry and the box size of the pure system were determined. Second, hydrogen and helium impurities were added, and the formation energies were computed while keeping the box size fixed. All atoms were fully relaxed until the change in energy upon ionic displacement was $<0.02 \mathrm{meV}$ and the forces were $<0.05 \mathrm{eV} / \AA$.

\section{II.C. Kinetic Monte Carlo Simulations}

In order to verify that the bubble formation results obtained from the DFTCs and MDSs have a bearing on the experimental findings of vastly different bubble depths for hydrogen and helium, we carried out KMCSs (Refs. 61 and 62) of long-time impurity atom migration, as well as atom self-trapping and defect-trapping. The general idea of KMCSs is to follow processes that occur with specified rates, like impurity atoms making diffusion jumps. KMC gives out the real time scale, which is not true for traditional Monte Carlo. The main advantage of KMCSs over MDSs is that the simulation time can be made much longer.

In short, the idea of our present use of KMCSs is as follows. In order to make bubble formation possible, the impurities must be allowed to trap either with each other or with defects, which can be native to the target or created by the irradiation. In order for the small bubbles formed in these ways to grow larger, one must assume-if there is no evidence- that the bubbles are able to grow continuously. We have previously shown that selftrapped helium bubbles indeed can grow in tungsten under continuous irradiation. ${ }^{49,50}$ For the KMCSs involving defects, we simply assume the presence of multiple filled traps, uniformly distributed in the target. These traps may be, e.g., grain boundaries or dislocations.

If the results from KMCSs using self-trapping agree with experiments, then an MDS prediction of a strongly bound $\mathrm{H}-\mathrm{H}$ or $\mathrm{He}-\mathrm{He}$ state provides the explanation that the configurational energetics of the small gas bubbles is the main cause responsible for the bubble formation. If the KMCSs do not agree with the experiments, then it is possible that self-trapping needs to be replaced or complemented with defect trapping.

For the study of self-trapping and defect trapping of hydrogen and helium in tungsten, a KMCS program for atom migration in an amorphous material was written. The free parameters in the input are the surface area (a square) of the target and the clustering radius (defined below); other parameters such as flux and temperature are taken from the literature. Experimentally determined 
migration prefactors and activation energies for hydrogen and helium were used. ${ }^{30,35}$ Initially, several square side lengths were tried. In the end, minimum lengths for which the bubble depths remained approximately constant were chosen for the high-fluence simulations. These were 2500 and $5000 \AA$ for helium, and 1000 and $4000 \AA$ for hydrogen.

When the distance between two atoms or a bubble and an atom is smaller than the clustering distance, the two entities are considered clustered. We used a clustering distance of $3.16 \AA$, equaling the lattice parameter of tungsten at zero kelvin. This value is reasonable for helium since our MDSs showed that two helium atoms less than $\approx 3 \AA$ from each other form a stable "dimer." In the case of hydrogen, the MDSs showed that two hydrogen atoms separated by $\approx 4 \AA$ or slightly less are attracted to one another. The lattice parameter (and jump lengths) change by $<2 \%$ when the temperature is increased to $3000 \mathrm{~K}$. This is quite a small increase, so the lengths at zero kelvin were used at all temperatures.

\section{RESULTS}

\section{III.A. Energy of Formation for a Hydrogen Pair in Tungsten}

An isolated interstitial hydrogen atom in tungsten occupies the tetrahedral $(T)$ site. ${ }^{63-66}$ A hydrogen atom at the tetrahedral site has four nearest-neighbor tungsten atoms, each at a distance of $1.91 \AA$. In the perfect hydrogen-free unrelaxed lattice, the corresponding distance is $1.77 \AA$. The tetrahedral geometry alone indicates there is some attraction rather than repulsion between the hydrogen and tungsten atoms; otherwise, the hydrogen atoms would probably occupy the octahedral $(O)$ site, which maximizes the distance to any neighboring lattice atom. Because of the tetrahedral geometry, there are four directional bonds between the hydrogen and the nearest tungsten atoms.

The potential energy $E_{p, \mathrm{H}-\mathrm{H}}$ of two hydrogen atoms at tetrahedral sites in tungsten are shown in Fig. 1. The potential energy is defined as $E_{p, \mathrm{H}-\mathrm{H}}(d)=E_{F}(d=\infty)-$ $E_{F}(d)$, where $E_{F}(d)$ is the formation energy of the hydrogen pair with an interatomic distance-separation - of $d$. With this definition $E_{p, \mathrm{H}-\mathrm{H}}=-E_{\text {bind }}$, where $E_{\text {bind }}$ is the binding energy of the hydrogen pair. Only stable states are shown in Fig. 1. The large number of points for distances of $4 \AA$ or larger is due to the greater number of available tetrahedral sites.

The $\mathrm{H}-\mathrm{H}$ configuration for which the separation is minimal, $\sim 0.72 \AA$, is the same as that for which the potential energy is the largest, $4.58 \mathrm{eV}$. The orientation of the $\mathrm{H}-\mathrm{H}$ bond is $(1,0.77,0.45)$. Each hydrogen atom has four almost equally long tungsten bonds: $2.29 \AA$ (or $2.28 \AA$ ) $, 2.29 \AA, 2.30 \AA$ (or $2.31 \AA$ ), and $2.34 \AA$ (or $2.33 \AA$ ). Putting a fictitious atom $\mathrm{X}$ at the center of mass

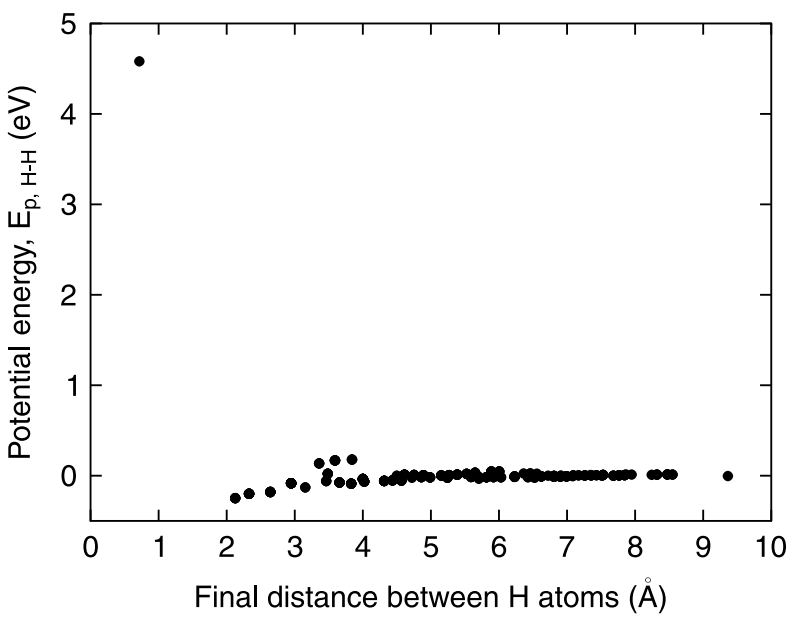

(a)

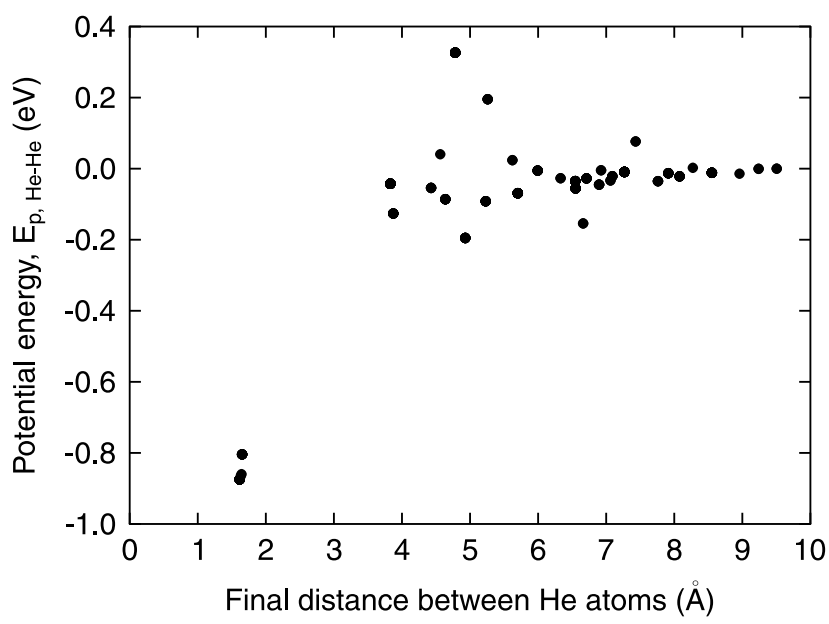

(b)

Fig. 1. Potential energy of (a) two hydrogen atoms at tetrahedral sites in tungsten and (b) two helium atoms at octahedral sites. The figure is the same as in our previous study (Ref. 53). The points above the curve in (a), for the interatomic distances $d \in(3,4) \AA$, differ from their neighboring $\left(d, E_{p, \mathrm{H}-\mathrm{H}}\right)$ points due to different angular configuration of the pair. The figure is the same as in our previous study (Ref. 53).

(c.m.) of the hydrogen pair and removing the hydrogen atoms, one finds that atom $\mathrm{X}$ has six tungsten bonds, but there are only three different bond lengths. This means that the c.m. of the hydrogen pair is located at a tetrahedral site, which is displaced toward the nearest octahedral site.

It should be noted that the distance of $0.72 \AA$ between the hydrogen atoms is very close to that of a free $\mathrm{H}_{2}$ molecule, which is $0.74 \AA$ (Ref. 67). If also the electronic configuration of the hydrogen pair resembles the cigar-like shape of the free $\mathrm{H}_{2}$ molecule, ${ }^{67}$ it helps to understand the high energy of formation: A relatively 
large amount of work is necessary to insert the extended $\mathrm{H}_{2}$ "cigar" into the tungsten lattice.

In the next stable $\mathrm{H}-\mathrm{H}$ configuration, the distance between the hydrogen atoms is $2.13 \AA$, and the potential energy is $-0.25 \mathrm{eV}$. The bond orientation is $(1, \sqrt{2} / 2,0)$.

Why do hydrogen atoms at tetrahedral sites with separations between 1.12 and $1.94 \AA$ not form stable pairs? The tetrahedral sites in question are labeled 1 through 4 in Fig. 2. The distances from site 1 to the three other tetrahedral sites are $d_{1-2}=a / 4 \cdot \sqrt{2}=1.12 \AA, d_{1-3}=$ $a / 2=1.58 \AA$, and $d_{1-4}=a / 4 \cdot \sqrt{6}=1.94 \AA$. In addition, the distance to the fifth tetrahedral site is $d_{1-5}=$ $a / 2 \cdot \sqrt{2}=2.23 \AA$. Thus, the stable hydrogen pair with next-to-smallest separation, $2.13 \AA$, is the $1-5$ configuration. One may argue that the 1-2, 1-3, and 1-4 config-

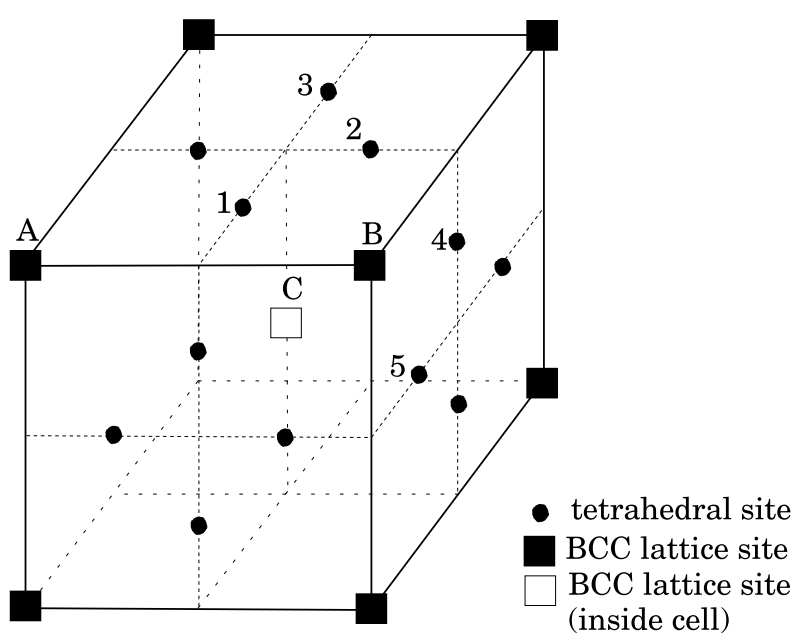

(a)

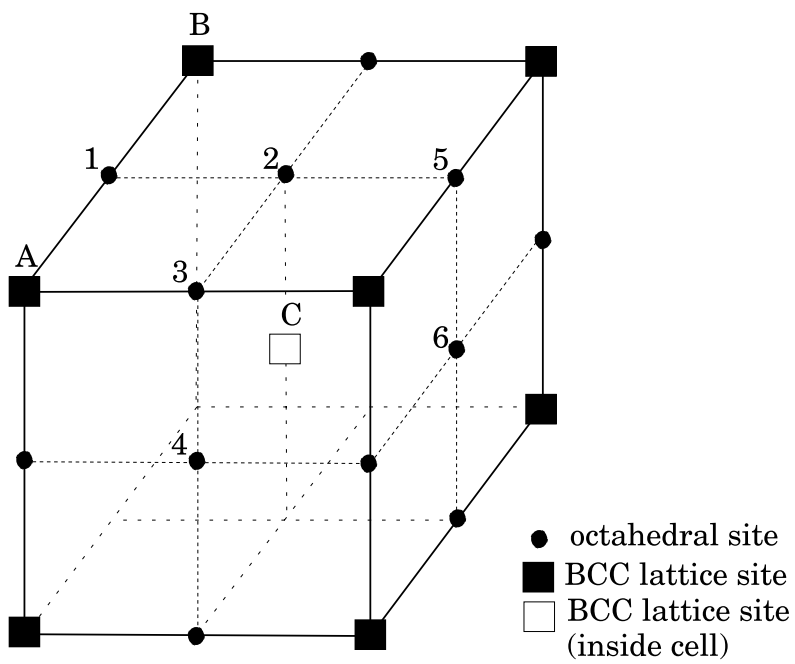

(b)

Fig. 2. Geometry of (a) tetrahedral and (b) octahedral sites in the BCC unit cell. urations are unstable since hydrogen atoms at these locations see too much of each other. At position 5, however, the tungsten lattice atom at site B screens the hydrogen atom at tetrahedral site 1 .

No consistent dependence of the formation energy on the mutual orientation of the $\mathrm{H}-\mathrm{H}$ separation vector was observed.

Density functional theory calculations of hydrogen in tungsten verify that when hydrogen is at a tetrahedral site, the total energy is lower than when hydrogen is at an octahedral site, the difference being $0.38 \mathrm{eV}$. The calculations show that $\mathrm{H}-\mathrm{H}$ pairs in tungsten are unstable since two hydrogen atoms introduced close to each other, at a separation of $d_{2}=1.59 \AA$, have a higher energy than two hydrogen atoms far apart, at a separation of $d_{3}=5.6 \AA$. The energy difference is $\left|E\left(d_{3}\right)-E\left(d_{2}\right)\right|=$ $0.53 \mathrm{eV}$. Also, relaxing the system with the hydrogen atoms fixed at a separation of $d_{1}=0.68 \AA$ gives an energy that is higher by $2.23 \mathrm{eV}$ (with respect to the configuration with a separation of $1.59 \AA$ ). This gives an energy $E\left(d_{1}\right)-E\left(d_{3}\right)=2.23-(-0.53)=2.76 \mathrm{eV}$ for this state, relative to two hydrogen atoms far apart. In summary, the DFTCs indicate that the hydrogen atoms in tungsten are unable to form stable pairs.

\section{III.B. Energy of Formation for a Helium Pair in Tungsten}

Helium atoms inserted into tungsten have been suggested to occupy the octahedral site..$^{35,68,69}$ Experimental data on the interstitial lattice location of helium in metals are scarce. However, atomistic simulations of helium atoms in several BCC metals (vandium, iron, molybdenum, and tungsten) indicate that the helium formation energy is indeed lower for the octahedral site; see Refs. 68, 70 , and references therein. The difference $E_{F, T}-E_{F, O}$ is $\sim 0.1$ to $0.2 \mathrm{eV}$. Our DFTC and MDS results indicate the octahedral and tetrahedral sites are within $0.3 \mathrm{eV}$ of each other, with the DFTCs predicting the tetrahedral site and the MDSs predicting the octahedral site, as the true ground state. This disagreement might be due to the small simulation box in the DFTCs. Also, the DFTCs converged to only $0.3 \mathrm{eV}$ for the energy difference between two helium atoms close to and far from each other. Therefore, the uncertainty in the DFTC energy values may be of this order. For the purpose of the present study, this difference in ground states - tetrahedral or octahedral-is not a significant problem, as will be argued below.

The octahedral site would be a natural place for the helium atom since at this position it maximizes the distance to any tungsten atom. For this configuration there are two nearest-neighbor tungsten atoms at a distance of $2.04 \AA$, the other four atoms being $2.29 \AA$ away, as predicted by MDSs. The corresponding distances in a perfect lattice are 1.58 and $2.23 \AA$, respectively. It would be qualitatively easy to understand why the helium atom occupies the octahedral site: Since helium has no valence electrons but a filled outer electron shell, there should not 
be any attractive interaction between helium and tungsten whatsoever.

The potential energy $E_{p, \mathrm{He}-\mathrm{He}}$ of two helium atoms at octahedral sites in tungsten are shown in Fig. 1. The smallest distance between two interstitial helium atoms in tungsten is $1.62 \AA$. The potential energy of this "dimer" state is $-0.87 \mathrm{eV}$. The orientation of the $\mathrm{He}-\mathrm{He}$ bond is (001) or (011). Each helium atom in this pair has six tungsten neighbors at a distance of $3.00 \AA$ or less, so that there are only three different bond lengths. The c.m. of the helium dimer is located at an octahedral site.

From the MDSs it is clear that helium atoms that are on octahedral sites corresponding to a pair of up to fourth nearest neighbors (distance between the helium atoms being $3.16 \AA$ ) form a strongly bound configuration (the relaxed distance between the helium atoms being $\sim 1.6 \AA$ ), but fifth nearest neighbors (distance of $3.53 \AA$ ) do not. This can be understood by considering the displacements of the repelled tungsten atoms and the effect they have on neighboring octrahedral sites. From Fig. 2 we have the distances $d_{1-2}=a / 2=1.58 \AA, d_{1-3}=$ $a / 2 \cdot \sqrt{2}=2.23 \AA, d_{1-4}=a / 2 \cdot \sqrt{2}=2.74 \AA, d_{1-5}=a=$ $3.16 \AA$, and $d_{1-6}=a / 2 \cdot \sqrt{5}=3.53 \AA$. First, tungsten atoms at positions $\mathrm{A}$ and $\mathrm{B}$ are repelled away from octahedral site 1 . The tungsten atom at site $\mathrm{C}$ and the three others at corresponding positions (not shown) are also repelled. But, any displacement away from octahedral site 1 along the line joining this site with the tungsten atom at site $\mathrm{C}$ will cause this atom to come closer to octahedral site 6 , making this site less suitable for a helium atom.

No consistent dependence of the formation energy on the mutual orientation of the He-He separation vector was observed.

DFTCs of helium in tungsten give $-1.30 \mathrm{eV}$ for the potential energy of the helium "dimer," with a distance of $1.53 \AA$ between the helium atoms. The corresponding distance in the MDSs is $1.62 \AA$. The energy result is in good agreement with that from the MDSs.

Above, it was mentioned that the different predictions of the MDSs and DFTCs about the ground state of helium in tungsten does not play any significant role. This is now clear since both MDSs and DFTCs predict a binding energy of $\sim 1 \mathrm{eV}$ for two close-by helium atoms.

\section{III.C. KMCSs of Hydrogen Implantation}

The three experimental studies of hydrogen implantation that were modeled with KMCSs are described in Table I. In the KMCSs the diffusion constant obtained by Frauenfelder was used; see Sec. I.B. The KMCS results for self-trapping are shown in Fig. 3 for the experimental setup of Haasz et al. ${ }^{8}$ The results for the other experiments are similar and therefore not shown. They were obtained for a square implantation area with the side length $1000 \AA$ A. These results were checked using a length of $6000 \AA$ and found to be similar. It is noteworthy that the bubble depth increases with the fluence and then decreases and stabilizes. This decrease and leveling out is due to the blocking of incident ions by the bubbles in the implantation region. The final depths of the selftrapped bubbles are given in Table II. In the following the bubble and trap depths are weighted with the occupancies (number of contained impurity atoms).

For the KMCSs using defect trapping, a box side length of $4000 \AA$ was used for all concentrations $c_{T}<$ $c_{T, 0}$ of traps capable of capturing multiple impurity atoms. These traps are called "multitraps" or "mtraps." For the original concentration $c_{T, 0}$, a box side length of $1000 \AA$ was used, except for the Wang et al. ${ }^{10}$ setup where a side length of $2000 \AA$ was employed. The original trap concentration was $c_{T, 0}=4.37 \times 10^{24} \mathrm{~m}^{-3}=4.37 \times$ $10^{18} \mathrm{~cm}^{-3}$. The corresponding value of $6.9 \times 10^{-5}$ traps/ tungsten was calculated by Anderl et al. ${ }^{71}$ from permeation data of a polycrystalline sample. We also found a value of $\sim 10^{-2}$ traps/tungsten in the literature, ${ }^{32}$ calculated from reemission data for single-crystalline and wrought samples. This latter value was not used since it seems quite high in comparison with the previous one. In addition to the original concentration $c_{T, 0}$, we also used concentrations $c_{T}=f c_{T, 0}$, with the fraction $f$ taking the values of $10^{-3}$ and $10^{-4}$. Plots of the occupancy of the traps and the trap depths as a function of the fluence are shown in Fig. 4 for the experimental setup of Haasz et al. The results for the Sze et al. setup are similar, but for Wang et al. the trap depths are much smaller (on the order of $100 \AA$ ), and the traps are not as heavily filled. The final depths of the multiple filled traps are given in Table III.

TABLE I

Experimental Parameters Used for the KMCSs of Hydrogen Implantation

\begin{tabular}{|c|c|c|c|c|c|c|c|}
\hline Experiment & Target & Isotope & $\begin{array}{c}\text { Temperature } \\
(\mathrm{K})\end{array}$ & $\underset{\left(m^{-2} s^{-1}\right)}{\text { Flux }}$ & $\begin{array}{c}\text { Ion } \\
\text { Energy } \\
(\mathrm{keV})\end{array}$ & $\begin{array}{c}\text { Fluence } \\
\left(\mathrm{m}^{-2}\right)\end{array}$ & $\begin{array}{l}\text { Bubble } \\
\text { Depth } \\
(\mu \mathrm{m})\end{array}$ \\
\hline Haasz et al. ${ }^{8}$ & Polycrystalline (99.96 wt\%) & Deuterium & 500 & $10^{20}$ & 0.500 & $10^{25}$ & 10 \\
\hline Sze et al. ${ }^{6}$ & Polycrystalline tungsten: $1 \% \mathrm{La}_{2} \mathrm{O}_{3}$ & Deuterium & 850 & $2 \times 10^{22}$ & 0.110 & $\geq 10^{26}$ & 5 and 10 \\
\hline Wang et al. ${ }^{10}$ & Polycrystalline (99.99 wt\%) & Deuterium & 300 & $1.1 \times 10^{20}$ & 1.000 & $10^{25}$ & 0.5 \\
\hline
\end{tabular}




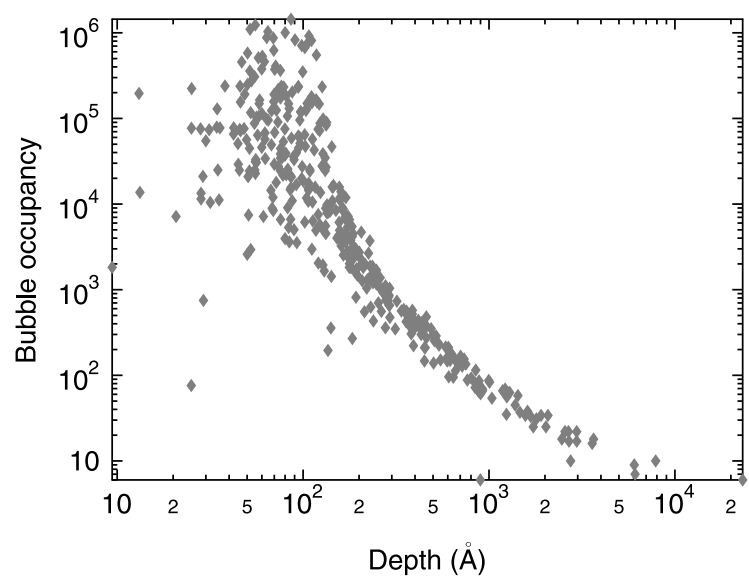

(a)

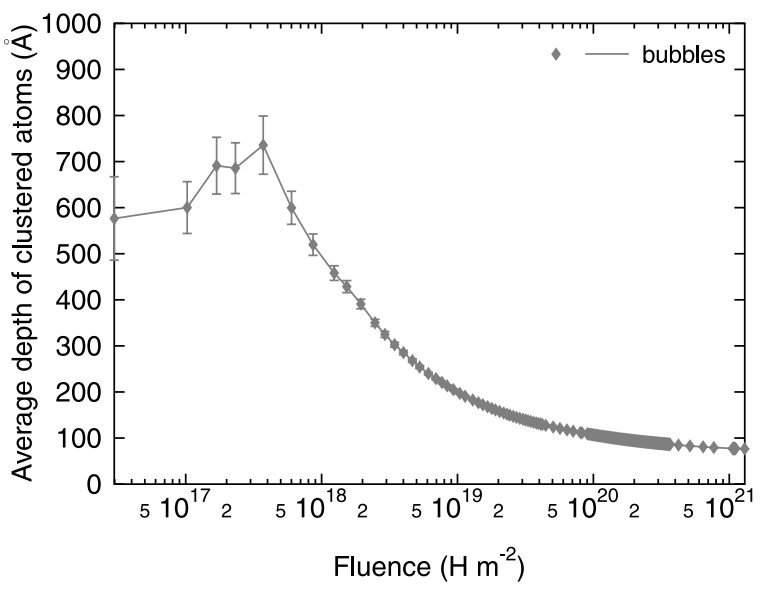

(b)

Fig. 3. Occupancy of self-trapped hydrogen bubbles at the maximum fluence reached in the KMCSs, and the average depth of the bubbles as a function of fluence. The results are for the Haasz et al. ${ }^{8}$ experimental setup. The results for the other setups are similar. The broken line is a guide for the eye.

To allow for extrapolation to lower concentrations, the trap depths were fitted to a power law $z_{m}=$ $a\left(c_{T} / c_{T, 0}\right)^{b}$. The fit gives $a=93.0 \pm 0.4, b=-0.388 \pm$ 0.002 for the Haasz et al. experiment and $a=58.3 \pm 0.3$,

TABLE II

Depths of Self-Trapped Hydrogen Bubbles

\begin{tabular}{|l|c|}
\hline Experiment & $\begin{array}{c}\text { Depth of Bubbles } \\
(\AA)\end{array}$ \\
\hline Haasz et al. & \\
Sze et al. & \\
Wang et al. & \\
& $76.50 \pm 0.01$ \\
& $36.23 \pm 0.02$ \\
& $112.40 \pm 0.05$ \\
\hline
\end{tabular}

TABLE III

Depths of Multiple Filled Hydrogen Traps, for Different Ratios $f$ of the Original Trap Concentration

\begin{tabular}{|c|c|c|c|}
\hline \multirow[b]{2}{*}{ Experiment } & \multicolumn{3}{|c|}{ Depth of Multiple Filled Traps $(\AA)$} \\
\hline & $f=1$ & $f=10^{-3}$ & $f=10^{-4}$ \\
\hline Haasz et al. ${ }^{8}$ & $93.0 \pm 0.4$ & $1410 \pm 20$ & $3160 \pm 60$ \\
\hline Sze et al. ${ }^{6}$ & $58.1 \pm 0.3$ & $1250 \pm 20$ & $2660 \pm 50$ \\
\hline Wang et al. ${ }^{10}$ & $133.3 \pm 0.4$ & $123 \pm 9$ & $154 \pm 10$ \\
\hline
\end{tabular}

$b=-0.426 \pm 0.002$ for the Sze et al. results. The fit to the Wang et al. results gave a very weakly falling line, but the uncertainty also permits a weakly rising line. The fits are shown in Fig. 5. These results indicate that the average depths of the multiple filled hydrogen traps in the Haasz et al. and Sze et al. experimental setups increase when the trap concentration is decreased. For the Wang et al. case, the results are not conclusive on the behavior of the trap depth.

\section{III.D. KMCSs of Helium Implantation}

The two experimental studies of helium implantation that were modeled with KMCSs are described in Table IV. The experimental diffusivity measured by Amano and Seidman ${ }^{35}$ was used.

The ion energy in the Chernikov and Zakharov ${ }^{20}$ experimental setup is very close to the threshold energy $503 \pm 12 \mathrm{eV}$ for lattice displacement. This value is calculated as follows. The maximum kinetic energy transferred to a lattice atom by the projectile in a collision is given by $E^{\prime}=4 m_{1} m_{2} /\left(m_{1}+m_{2}\right)^{2} E$, where $E$ is the energy of the incident atom and $m_{1}, m_{2}$ are the masses of the lattice atom and the projectile, respectively. ${ }^{35}$ For ${ }_{2}^{4} \mathrm{He}\left(m_{1}=4.0026 \mathrm{u}\right)$ and the average tungsten atom $\left(m_{2}=183.84 \mathrm{u}\right)$ (see Ref. 38), and the maximum energy $E^{\prime}$ equal to the lattice displacement threshold energy of $42 \pm 1 \mathrm{eV}$ [in the (100) direction; see Ref. 72], the minimum helium energy for damage production is $E=503 \pm$ $12 \mathrm{eV}$. Because of the closeness of the ion energy value of $510 \mathrm{eV}$ to the threshold energy, we first checked with SRIM (Ref. 27) the amount of defects created. The results showed that $<5 \times 10^{-5}$ vacancies were created by each ion. Because of the very low value, we assumed only self-trapping when simulating this experimental setup.

The results for the Chernikov and Zakharov ${ }^{20}$ setup are shown in Fig. 6, for a square side length of $5000 \AA$. The final depths of the self-trapped helium bubbles are presented in Table $\mathrm{V}$.

Since the 1-keV ion energy used by Nicholson and Walls ${ }^{18}$ causes lattice damage in tungsten, this setup was simulated using both self-trapping and defect trapping. The 


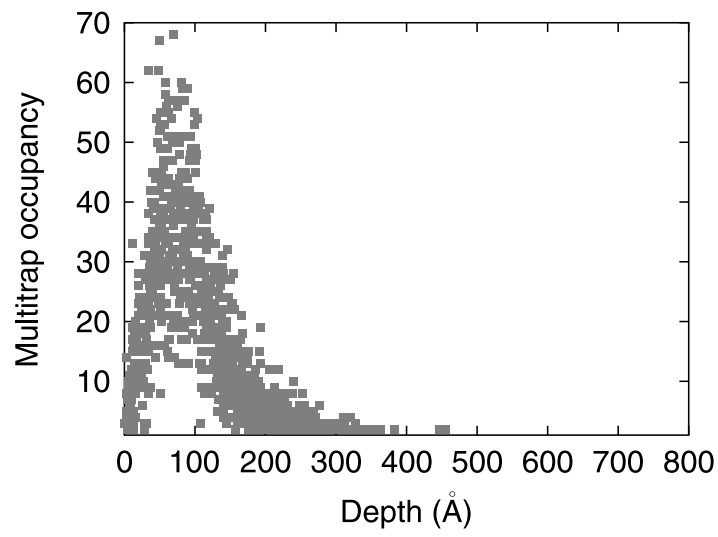

(a)

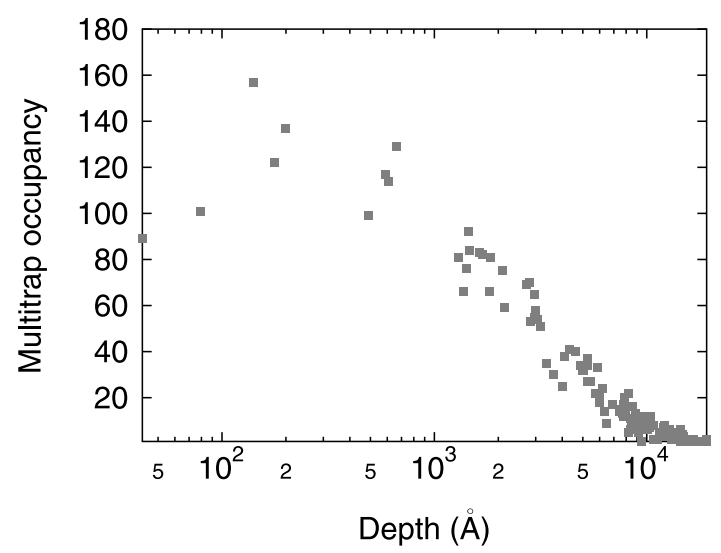

(c)

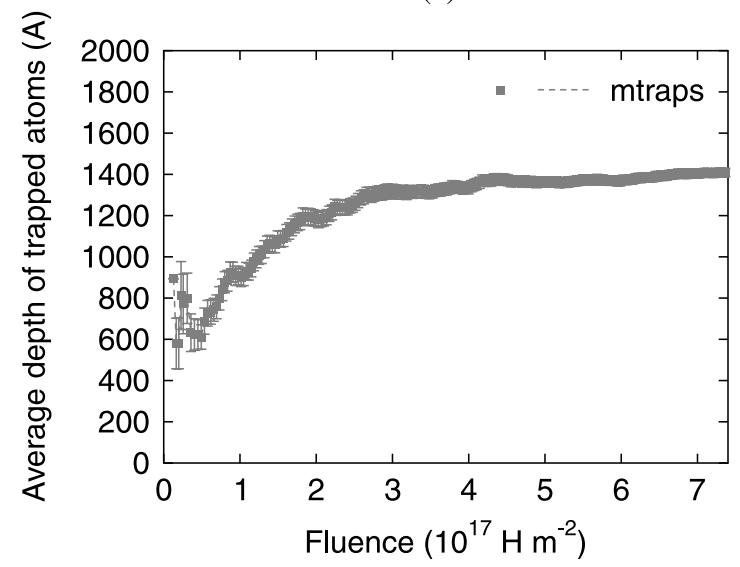

(e)

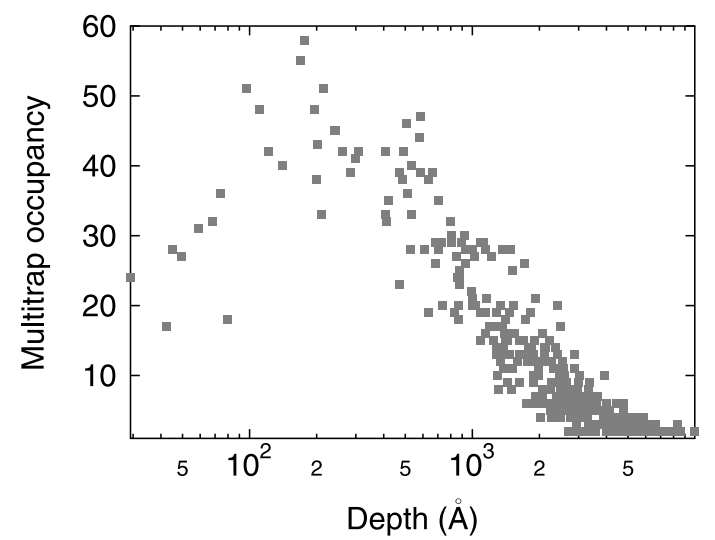

(b)

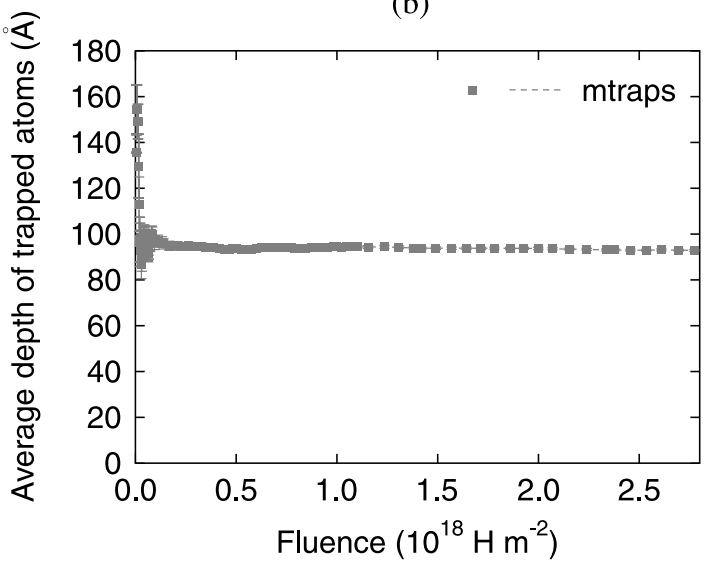

(d)

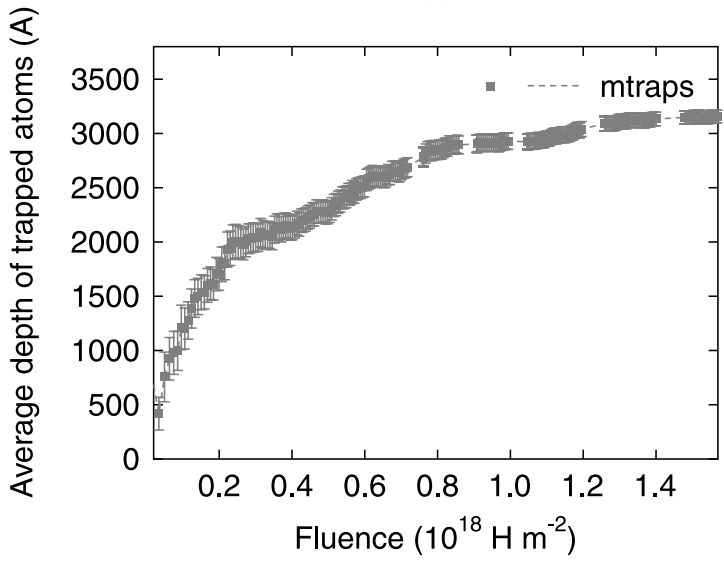

(f)

Fig. 4. Occupancy of multiple filled hydrogen traps at the maximum fluence reached in the KMCSs, and the average depth of the traps as a function of fluence. The results are for the Haasz et al. ${ }^{8}$ experimental setup, using a defect concentration of $f c_{T, 0}$, with (a) $f=1$, (b) $f=10^{-3}$, and (c) $f=10^{-4}$. The label "mtraps" stands for multiple filled traps. The broken line is a guide for the eye.

present defects are different from those used in the KMCSs for hydrogen in tungsten. Instead of uniformly distributed native defects, the present defects are radiation induced, namely, vacancies. The defect distributions were obtained from SRIM calculations. A square side length of $2500 \AA$ was used in the KMCSs. The results are shown in Fig. 7. The final depths of the self-trapped helium bubbles and multiple filled helium traps are in Table V. 


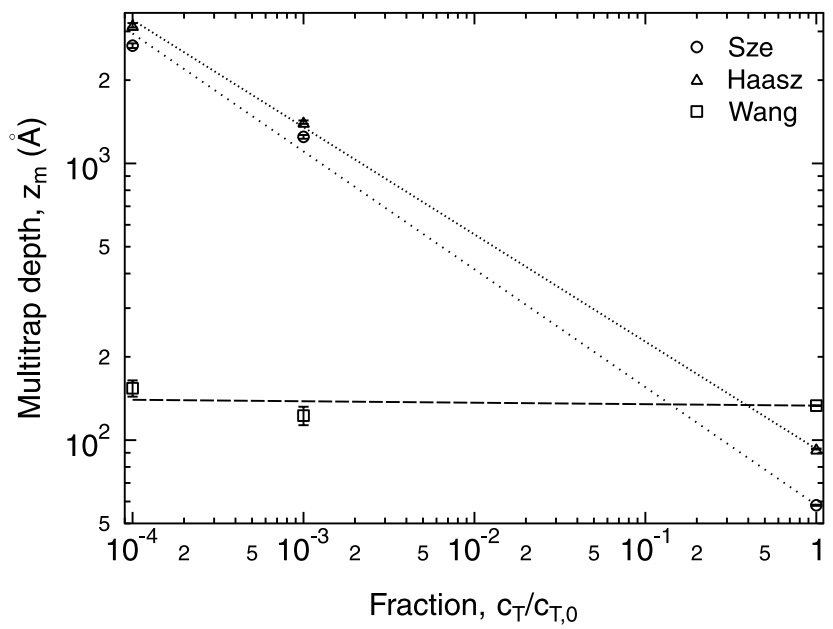

Fig. 5. Depth of multiple filled hydrogen traps as a function of the defect concentration $c_{T}$ normalized to the value $c_{T, 0}$ in the literature.

\section{DISCUSSION}

\section{IV.A. Energy of Formation for a Hydrogen and Helium Pair in Tungsten}

Our MDS and DFTC results on the binding energy of hydrogen and helium pairs in tungsten (Secs. III.A and III.B) can be summarized as follows. It is extremely difficult for two hydrogen atoms to form a covalently bonded state. Instead, it may form a loosely bound pair (binding energy $0.25 \mathrm{eV}$ from MDSs) at an interatomic separation of $\sim 2 \AA$. Helium atoms, on the other hand, do form pairs with a binding energy of $0.87 \mathrm{eV}$. These binding energies imply that at room temperature hydrogen atoms in practice do not bind with other hydrogen atoms, while helium atoms do. Assuming a breakup rate of the pairs of the form $w(T)=w_{0} \exp \left[-E_{b} /\left(k_{B} T\right)\right]$, with $E_{b}$ the binding energy, $k_{B}$ Boltzmann's constant, and $T$ the temperature, one has $w_{\mathrm{H}} / w_{\mathrm{He}} \sim \exp \left[-(0.25-0.87) \mathrm{eV} /\left(k_{B} 300 \mathrm{~K}\right)\right] \sim$ $\exp [26.0] \gg 1$.

When presenting the MDS results for the potential energy curve of hydrogen and helium in tungsten, we motivated the various configurations using arguments

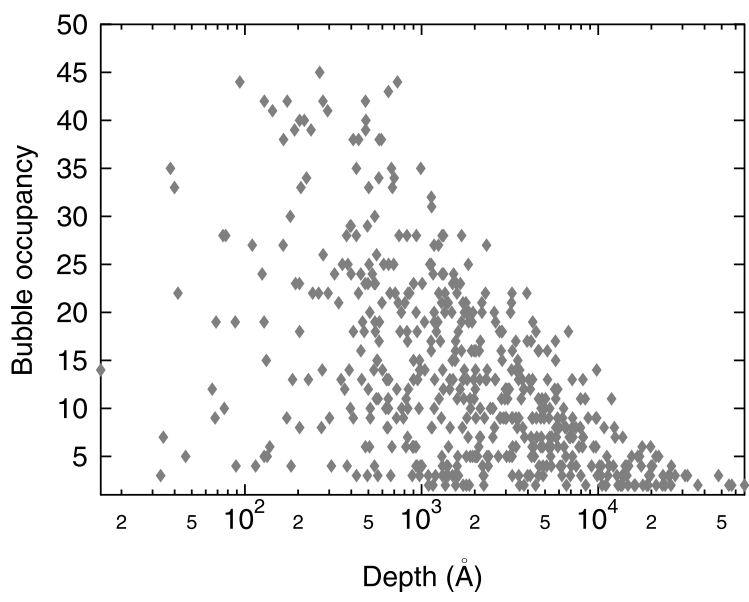

(a)

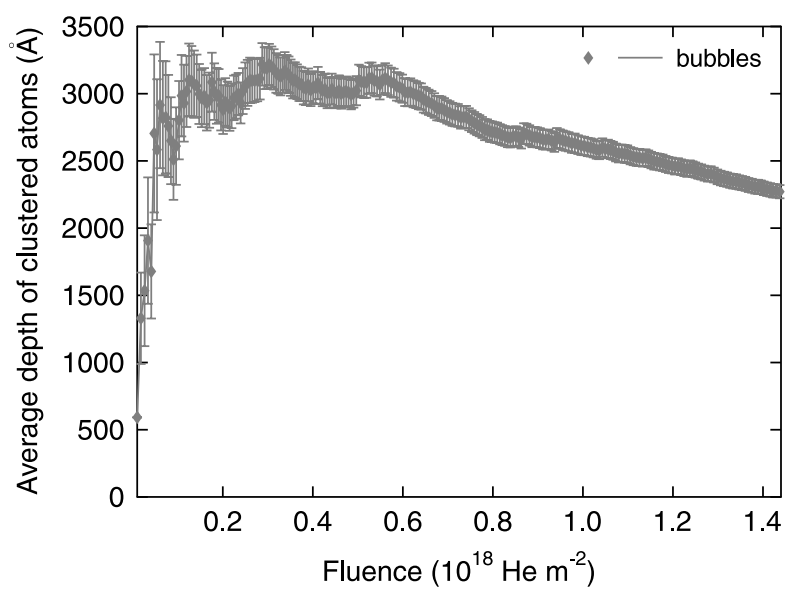

(b)

Fig. 6. Occupancy of self-trapped helium bubbles at the maximum fluence reached in the KMCSs, and the average depth of the bubbles as a function of fluence, for the Chernikov and Zakharov ${ }^{20}$ experimental setup. The broken line is a guide for the eye.

based on electronic interaction. It should be noted that our MDSs do not include any explicit calculations of electronic structure or charge transfer, so we have no direct evidence to verify our arguments. However, the

TABLE IV

Experimental Parameters Used for the KMCSs of Helium Implantation

\begin{tabular}{|c|c|c|c|c|c|c|}
\hline \multicolumn{1}{|c|}{ Experiment } & \multicolumn{1}{|c|}{ Target } & $\begin{array}{c}\text { Temperature } \\
(\mathrm{K})\end{array}$ & $\begin{array}{c}\text { Flux } \\
\left(\mathrm{m}^{-2} \mathrm{~s}^{-1}\right)\end{array}$ & $\begin{array}{c}\text { Ion } \\
\text { Energy } \\
(\mathrm{keV})\end{array}$ & $\begin{array}{c}\text { Fluence } \\
\left(\mathrm{m}^{-2}\right)\end{array}$ & $\begin{array}{c}\text { Bubble } \\
\text { Depth } \\
(\AA)\end{array}$ \\
\hline $\begin{array}{l}\text { Chernikov and Zakharov } \\
\text { Nicholson and Walls } 18\end{array}$ & $\begin{array}{l}\text { Single crystalline }(99.99 \mathrm{wt} \%) \\
\text { Single crystalline }\end{array}$ & $\begin{array}{c}2370 \pm 50 \\
300\end{array}$ & $\begin{array}{c}1.124 \times 10^{22} \\
1.2 \times 10^{18}\end{array}$ & $\begin{array}{c}0.510 \\
1.0\end{array}$ & $\begin{array}{c}4.045 \times 10^{25} \\
5 \times 10^{20}\end{array}$ & $\begin{array}{c}0 \text { to } 5000 \\
62\end{array}$ \\
\hline
\end{tabular}


TABLE V

Depths of Self-Trapped Helium Bubbles and Multiple Filled Helium Traps

\begin{tabular}{|l|c|c|c|}
\hline & $\begin{array}{c}\text { Depth } \\
\text { of } \\
\text { Bubbles } \\
(\AA)\end{array}$ & $\begin{array}{c}\text { Depth } \\
\text { of } \\
\text { Multiple } \\
\text { Filled } \\
\text { Traps } \\
\AA\end{array}$ & $\begin{array}{c}\text { Depth } \\
\text { of } \\
\text { Bubbles } \\
\text { and } \\
\text { Traps } \\
(\AA)\end{array}$ \\
\hline $\begin{array}{l}\text { Experiment } \\
\text { Chernikov and Zakharov } 20\end{array}$ & $\begin{array}{c}2260 \pm 50 \\
\text { Nicholson and Walls }\end{array}$ & - & - \\
\hline
\end{tabular}

configurations calculated with DFTCs-which make direct use of electronic states-do provide evidence of a repulsion between two close-by hydrogen atoms and, perhaps, suggest an electrostatic repulsion between hydrogen atoms removed of their electrons. In defense of the electron-configuration interpretations made from the MDS results of the $\mathrm{H}-\mathrm{H}$ states, we want to point out that the potentials have angular-dependent components and make use of electron densities.

Explicit elastic interaction energies of two hydrogen atoms in tungsten (and other BCC metals) have been calculated by Blanter ${ }^{73}$ using the Khachaturyan theory of lattice statics. It was found that the elastic straininduced interaction between hydrogen atoms in tungsten is attractive at distances $<2.7 \AA$, the potential energy being of the order of $-0.1 \mathrm{eV}$ at distances $d \in[1.1,2.5] \AA$. These energies were calculated using pair interactions; any negative or positive contribution due to angular dependence is not included. In addition to the attraction, there should be some repulsion at small interatomic distances, but this was not calculated for tungsten. From the data for vanadium, niobium, and tantalum, one may perhaps estimate at which interatomic distance the attraction turns into a repulsion in tungsten. Using tantalum as the closest approximation to tungsten (since these are adjacent elements on the same row in the periodic system), one obtains that the energy of elastic attraction is largest at $d=1.93 \AA$. This compares favorably to our MDS results, which indicate that $d$ is $2.13 \AA$ (see Fig. 1). It also agrees partly with our DFTC findings, according to which a $\mathrm{H}-\mathrm{H}$ pair with a separation of $1.59 \AA$ is unstable.

\section{IV.B. KMCSs of Implantation}

\section{IV.B.1. Hydrogen}

The KMCS results for hydrogen implanted into polycrystalline tungsten clearly indicate that if hydrogen atoms are able to self-trap when the interatomic distance is $3.16 \AA$ or less, the bubbles will form much more closely to the surface than has been observed in experiments. These results indicate that self-trapping of hydrogen should be

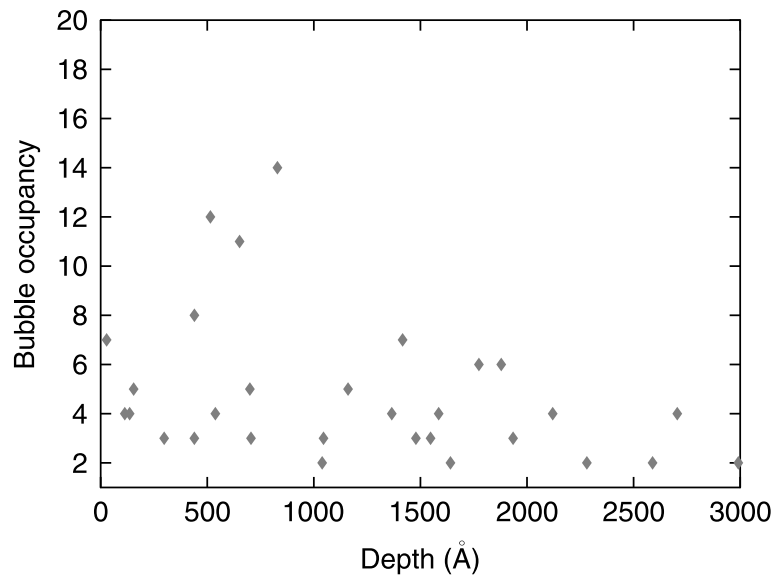

(a)

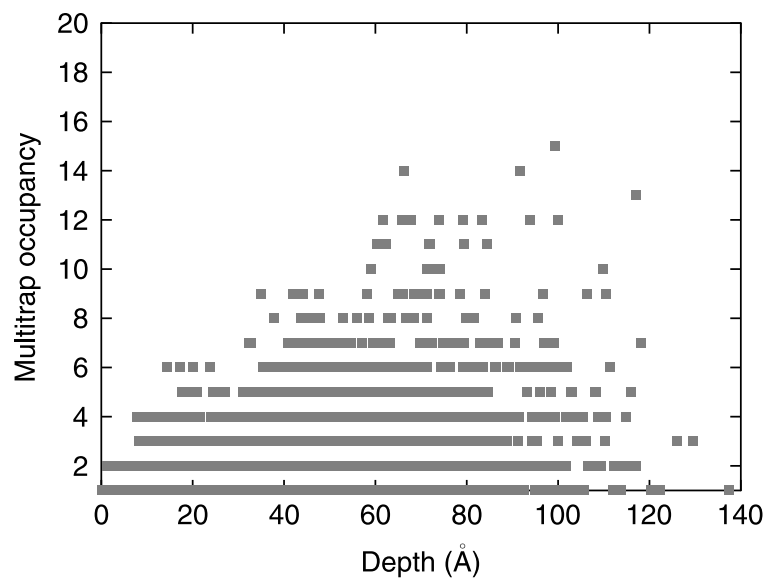

(b)

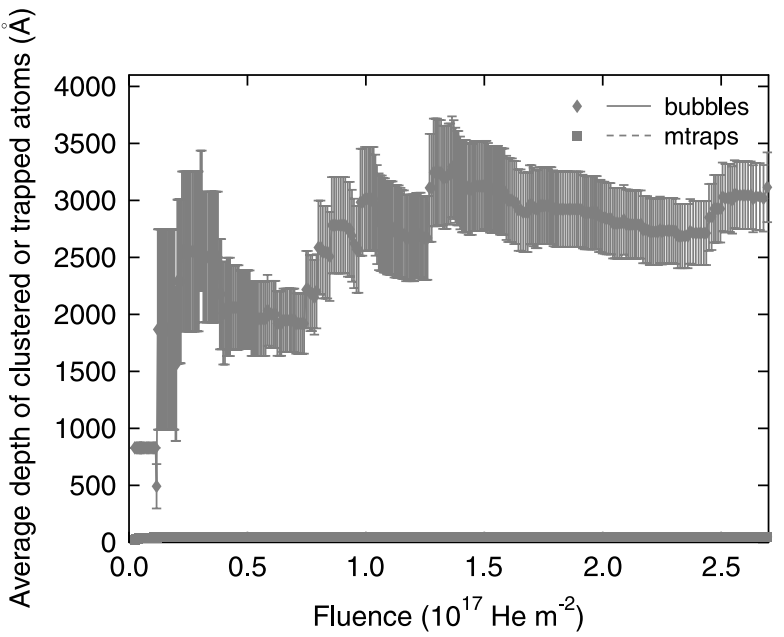

(c)

Fig. 7. Occupancy of (a) self-trapped helium bubbles and (b) multiple filled helium traps at maximum fluence reached in the KMCSs, as well as (c) the average depths of these as a function of fluence, for the Nicholson and Walls ${ }^{18}$ experiment. The label "mtraps" stands for multiple filled traps. The broken line is a guide for the eye. 
weak in polycrystalline tungsten and that the main mechanism for bubble formation must be sought elsewhere. This agrees with our DFT and MDS results showing that hydrogen traps only weakly with itself.

It has been observed in experiments that nondamaging deuterium irradiation with a flux of $\sim 10^{22}$ ions $\mathrm{m}^{2} \mathrm{~s}^{-1}$ up to a fluence of $\geq 10^{25}$ ions $\mathrm{m}^{-2}$ of single-crystalline tungsten creates blisters with diameters 1 to $2 \mu \mathrm{m}$ on a (100) surface at $343 \mathrm{~K}$ but not at $\geq 383 \mathrm{~K}$ (Ref. 51 ) and that no blisters are found on a (111) surface at $770 \mathrm{~K}$ (Ref. 52). These results indicate that high flux and fluence are not enough to produce blisters in single-crystalline tungsten; a low temperature is also needed. The high implantation rate and low escape rate of deuterium ions could make the formation of weakly bound hydrogen pairs in the implantation zone possible. But, this would result in surface-near clusters, and the mechanism by which these are converted into blisters is not evident. Traps such as impurities and dislocations could perhaps also be responsible for the buildup of small hydrogen clusters. Another possibility is that the blisters are created due to buildup of lateral stresses. In any case, more studies of blister formation in single-crystalline tungsten under nondamaging irradiation are needed to fully explain how they come about.

The experimentally determined bubble depths are larger than those obtained in our KMCSs, even with a trap concentration that is $10^{-4}$ of the one taken from the literature. From the dependence $z_{m}=a f^{b}$ for the depth of defect-trapped bubbles, we can estimate which fraction $f=c_{T} / c_{T, 0}$ of the original defect concentration is needed to obtain simulated bubbles at the experimental depths. Sze et al. ${ }^{6}$ observed hydrogen bubbles at depths of 5 and $10 \mu \mathrm{m}$. This corresponds to a fraction $f \sim 10^{-8} \ldots 10^{-7}$. Haasz et al. ${ }^{8}$ observed hydrogen bubbles at depths of $10 \mu \mathrm{m}$. This implies a fraction $f \sim 10^{-8}$. The necessary defect concentration becomes $c_{T} \sim 10^{16}$ traps $\mathrm{m}^{-3}=$ $10^{10}$ traps $\mathrm{cm}^{-3}$ or $\sim 10^{-12}$ traps/tungsten. It should be remembered we have assumed a simple power law behavior $z_{m} \propto f^{b}$ for the defect-trapped bubbles, so these values for $f$ are only rough estimates. A similar prediction cannot be made for the results obtained by using the experimental setup of Wang et al. ${ }^{10}$ since a conclusive $z_{m}$ dependence on $f$ was not obtained.

There are two possible reasons for the discrepancy between the experimental results and the predictions from our KMCSs concerning the depths of hydrogen bubbles. First, all the experimental studies that were modeled with KMCSs used polycrystalline tungsten samples, with a purity of $99.99 \mathrm{wt} \%$ or less, and in most cases the samples were not annealed. In the study by Sze et al., ${ }^{6}$ the samples contained $1 \% \mathrm{La}_{2} \mathrm{O}_{3}$ and were used as-received, without annealing. The only experiment where annealing was carried out was that of Haasz et al. ${ }^{8}$ In this study the samples were annealed for $1 \mathrm{~h}$, at a temperature close to or in excess of $1473 \mathrm{~K}$. In the study by Wang et al. ${ }^{10}$ the samples were outgassed at $1123 \mathrm{~K}$ for $15 \mathrm{~min}$. It is pos- sible the lack of annealing treatment in two of these studies ${ }^{6,10}$ could have left vacancy-rich grain boundaries in the samples. Furthermore, it is likely that hydrogen atoms migrate faster along "spacious" grain boundaries since they encounter fewer host atoms. Therefore, it may be that hydrogen atoms actually migrated faster in the experiments than in the simulations, therefore resulting in more shallow simulated bubble depths. As a matter of fact, Komarov et al. ${ }^{74}$ have investigated deuterium retention in polycrystalline tungsten with a purity of $99.94 \%$, annealed at $800 \mathrm{~K}$ for $10 \mathrm{~min}$, and single-crystalline tungsten. The samples were subjected to deuterium plasma at $500 \mathrm{~K}$ for the same amount of time. The retention analysis showed that the polycrystalline sample contained deuterium in the bulk region (down to a depth of $4 \mu \mathrm{m}$ ), whereas no deuterium was found in the single-crystalline sample, down to $2 \mu \mathrm{m}$. Komarov et al. concluded that there must have been a significant transport of deuterium along the grain boundaries.

Second, it is reasonable to assume that detrapping events lead to deeper average bubble depths at elevated temperatures. In our KMCSs, detrapping was not considered. In order to fully account for this type of event, the binding energy of multiple filled traps as a function of occupancy would have to be known. This type of datum exists for small clusters, consisting of 1 to 2 hydrogen atoms in a vacancy ${ }^{75}$; but without some growth mechanism that actually increases the size of the cavity (from one vacancy), these clusters cannot account for the large bubbles. In the literature there are reports of traps with energies between $0.5 \mathrm{eV}$ ("natural traps" in unirradiated single-crystalline tungsten ${ }^{32}$ ) and $1.6 \mathrm{eV}$ (traps in an unirradiated and unannealed polycrystalline tungsten ${ }^{72}$ ), but the dependence of the binding energies on the hydrogen occupancy has not been reported. This makes it difficult to incorporate detrapping behavior into more detailed KMCSs.

\section{IV.B.2. Helium}

In the experiment by Nicholson and Walls ${ }^{18}$ using 1-keV helium ions, bubbles were observed at a depth of $62 \AA$ after a fluence of $5 \times 10^{16}$ ions $\mathrm{m}^{-2}$. Our KMCSs give a depth of defect-trapped bubbles of $\sim 49 \AA$, a depth of self-trapped bubbles of $\sim 3100 \AA$, and an average depth in the range of 100 to $300 \AA$ of both bubbles and filled traps. In light of this last value, our results agree with those of Nicholson and Walls.

It should also be noted that the experimental bubble depths were obtained by removing successive atomic layers by FIM. Therefore, it is possible that experimental regions deeper than say $200 \AA$ were not analyzed at all. If this was the case, then 3100 - $\AA$ deep bubbles like the ones observed in our KMCSs could have been present in the experimental target. Also, our results show that the bubbles are not as many or as dense as the traps, which may explain why these were less visible or obvious in the experiment. 
The bubbles observed by Chernikov and Zakharov ${ }^{20}$ were situated at depths in the range 0 to $5000 \AA$. Our results of self-trapped bubbles at $\sim 2300 \AA$ are in good agreement with these.

\section{CONCLUSIONS}

We have provided an explanation for the vastly different depths of hydrogen and helium bubbles found in experiments on tungsten after nondamaging ion irradiation. The fundamental reason is the different self-trapping behavior of the gas ions in the target. Our DFTCs and MDSs show that two hydrogen atoms trap each other weakly with a binding energy of $<0.3 \mathrm{eV}$, whereas helium atoms form strong pairs with a binding energy of $\sim 1 \mathrm{eV}$. Because of this, larger helium bubbles can form spontaneously close to the projected range, whereas hydrogen atoms migrate deeply down into the tungsten target before becoming trapped by defects, as verified by the present results of KMCSs. These findings are also relevant to blistering, as they help to explain why the lid thicknesses of hydrogen blisters are so much larger than the projected range of the ions.

\section{ACKNOWLEDGMENTS}

Financial support from Association Euratom-Tekes and the Magnus Ehrnrooth foundation is gratefully acknowledged.

\section{REFERENCES}

1. B. M. U. SCHERZER, Development of Surface Topography due to Gas Ion Implantation, in Sputtering by Particle Bombardment, Vol. II, Chap. 7, p. 271, Springer-Verlag, Berlin, Germany (1983).

2. R. A. CAUSEY and T. J. VENHAUS, Phys. Scripta, T94, 9 (2001).

3. W. PRIMAK, Y. DAYAL, and E. EDWARDS, J. Appl. Phys., 34, 827 (1963).

4. W. PRIMAK, J. Appl. Phys., 34, 3630 (1963).

5. R. SAKAMOTO, T. MUROGA, and N. YOSHIDA, J. Nucl. Mater., 220-222, 819 (1995).

6. F. C. SZE, R. P. DOERNER, and S. LUCKHARDT, J. Nucl. Mater., 264, 89 (1999).

7. F. C. SZE, L. CHOUSAL, R. P. DOERNER, and S. LUCKHARDT, J. Nucl. Mater., 266-269, 1212 (1999).

8. A. A. HAASZ, M. POON, and J. W. DAVIS, J. Nucl. Mater., 266-269, 520 (1999).

FUSION SCIENCE AND TECHNOLOGY VOL. $50 \quad$ JULY 2006
9. T. VENHAUS, R. CAUSEY, R. DOERNER, and T. ABELN, J. Nucl. Mater., 290-293, 505 (2001).

10. W. WANG, J. ROTH, S. LINDIG, and C. H. WU, J. Nucl. Mater., 299, 124 (2001).

11. K. TOKUNAGA, R. P. DOERNER, R. SERAYDARIAN, N. NODA, N. YOSHIDA, T. SOGABE, T. KATO, and B. SCHEDLER, J. Nucl. Mater., 307-311, 126 (2002).

12. M. Y. YE, H. KANEHARA, S. FUKUTA, N. OHNO, and S. TAKAMURA, J. Nucl. Mater., 313-316, 72 (2003).

13. T. SHIMADA, H. KIKUCHI, Y. UEDA, A. SAGARA, and M. NISHIKAWA, J. Nucl. Mater., 313-316, 204 (2003).

14. D. NISHIJIMA, T. SUGIMOTO, M. YE, N. OHNO, and S. TAKAMURA, Jpn. J. Appl. Phys., 44, 380 (2005).

15. Y. UEDA, T. FUNABIKI, T. SHIMADA, K. FUKUMOTO, H. KURISHITA, and M. NISHIKAWA, J. Nucl. Mater. 337-339, 1010 (2005).

16. D. NISHIJIMA, H. IWAKIRI, K. AMANO, M. Y. YE, N. OHNO, K. TOKUNAGA, N. YOSHIDA, and S. TAKAMURA, Nucl. Fusion, 45, 669 (2005).

17. J. M. WALLS, R. M. BOOTHBY, and H. N. SOUTHWORTH, Surface Sci., 61, 419 (1976).

18. R. J. K. NICHOLSON and J. M. WALLS, J. Nucl. Mater., 76-77, 251 (1978).

19. N. YOSHIDA, E. KURAMOTO, and K. KITAJIMA, J. Nucl. Mater., 103-104, 373 (1981).

20. V. N. CHERNIKOV and A. P. ZAKHAROV, J. Nucl. Mater., 165, 89 (1989).

21. H. IWAKIRI, K. YASUNAGA, K. MORISHITA, and N. YOSHIDA, J. Nucl. Mater., 283-287, 1134 (2000).

22. S. NAGATA, B. TSUCHIYA, T. SUGAWARA, N. OHTSU, and T. SHIKAMA, Nucl. Instrum. Methods Phys. Res. B, 190, 652 (2002).

23. D. NISHIJIMA, M. Y. YE, N. OHNO, and S. TAKAMURA, J. Nucl. Mater., 313-316, 97 (2003).

24. D. NISHIJIMA, M. Y. YE, N. OHNO, and S. TAKAMURA, J. Nucl. Mater., 329-333, 1029 (2004).

25. D. NISHIJIMA, T. SUGIMOTO, H. IWAKIRI, M. Y. YE, N. OHNO, N. YOSHIDA, and S. TAKAMURA, J. Nucl. Mater., 337-339, 927 (2005).

26. M. MIYAMOTO, M. TOKITANI, K. TOKUNAGA, F. FUJIWARA, N. YOSHIDA, S. MASUZAKI, and A. KOMORI, J. Nucl. Mater., 329-333, 742 (2004).

27. J. F. ZIEGLER, "SRIM-2003 Software Package"; available online at $\langle$ http://www.srim.org $\rangle$.

28. G. E. MOORE and F. C. UNTERWALD, J. Chem. Phys., 40, 2639 (1964). 
29. L. N. RYABCHIKOV, Ukr. Fiz. Zh. (Ukr. Phys. J.), 9, 293 (1964)

30. R. FRAUENFELDER, J. Vac. Sci. Technol., 6, 388 (1969).

31. A. P. ZAKHAROV, V. M. SHARAPOV, and E. I. EVKO, Fiz-Khim. Mekh. Mater., 9, 29 (1973).

32. P. FRANZEN, C. GARCIA-ROSALES, H. PLANK, and V. K. ALIMOV, J. Nucl. Mater., 241-243, 1082 (1997).

33. G. A. EStebAn, A. PERUJO, L. A. SEDANO, and K. DOUGLAS, J. Nucl. Mater., 295, 49 (2001).

34. R. A. CAUSEY, J. Nucl. Mater., 300, 91 (2002).

35. J. AMANO and D. N. SEIDMAN, J. Appl. Phys., 56, 983 (1984).

36. A. T. MACRANDER and D. N. SEIDMAN, J. Appl. Phys., 56, 1623 (1984).

37. H. WIPF, Hydrogen in Metals III, in Topics in Applied Physics, Vol. 73, Chap. 3, p. 51, Springer-Verlag, Berlin, Germany (1997).

38. D. R. LIDE, CRC Handbook of Chemistry and Physics, 82nd ed., CRC Press, Boca Raton, Florida (2001).

39. R. BEHRISCH, J. BOTTIGER, W. ECKSTEIN, U. LITTMARK, J. ROTH, and B. M. U. SCHERZER, Appl. Phys. Lett., 27, 199 (1975).

40. E. P. EERNISSE and S. T. PICRAUX, J. Appl. Phys., 48, 9 (1977).

41. Y. V. MARTYNENKO, Radiat. Effects, 45, 93 (1979).

42. S. T. PICRAUX, Nucl. Instrum. Methods, 182/183, 413 (1981).

43. A. C. SWITENDICK, Hydrogen in Metals I, in Topics in Applied Physics, Vol. 28, Chap. 5, p. 101, Springer-Verlag, Berlin, Germany (1978).

44. J. K. NØRSKOV, Phys. Rev. B, 20, 446 (1979).

45. R. B. McLELLAN, Scripta Metall., 9, 681 (1975).

46. J. K. NØRSKOV, F. BESENBACHER, J. BOTTIGER, B. B. NIELSEN, and A. A. PISAREV, Phys. Rev. Lett., 49, 1420 (1982).

47. P. NORDLANDER, J. K. NØRSKOV, and F. BESENBACHER, J. Phys. F: Met. Phys., 16, 1161 (1986).

48. J. K. NØRSKOV, Europhysics News, 19, 65 (1988).

49. K. O. E. HENRIKSSON, K. NORDLUND, J. KEINONEN, D. SUNDHOLM, and M. PATZSCHKE, Phys. Scripta, T108, 95 (2004).

50. K. O. E. HENRIKSSON, K. NORDLUND, and J. KEINONEN, Nucl. Instrum. Methods Phys. Res. B, 244, 377 (2006).
51. K. TOKUNAGA, M. J. BALDWIN, R. P. DOERNER, N. NODA, Y. KUBOTA, N. YOSHIDA, T. SOGABE, T. KATO, and B. SCHEDLER, J. Nucl. Mater., 337-339, 887 (2005).

52. M. I. GUSEVA et al., J. Nucl. Mater., 290-293, 1069 (2001).

53. K. O. E. HENRIKSSON, K. NORDLUND, A. KRASHENINNIKOV, and J. KEINONEN, Appl. Phys. Lett., 87, 163113 (2005).

54. G. J. ACKLAND and R. THETFORD, Phil. Mag. A, 56, 15 (1987).

55. J. F. ZIEGLER, J. P. BIERSACK, and U. LITTMARK, The Stopping and Range of Ions in Matter, Pergamon, New York (1985).

56. N. JUSLIN, J. NORD, K. O. E. HENRIKSSON, P. TRÄSKELIN, E. SALONEN, K. NORDLUND, P. ERHART, and K. ALBE, J. Appl. Phys., 98, 123520 (2005).

57. V. MILMAN, B. WINKLER, J. A. WHITE, C. J. PICKARD, M. C. PAYNE, E. V. AKHMATSKAYA, and R. H. NOBES, Int. J. Quant. Chem., 77, 895 (2000).

58. J. P. PERDEW, J. A. CHEVARY, S. H. VOSKO, K. A. JACKSON, M. R. PEDERSON, D. J. SINGH, and C. FIOLHAIS, Phys. Rev. B, 46, 6671 (1992).

59. G. KRESSE and J. JOUBERT, Phys. Rev. B, 59, 1758 (1999).

60. P. E. BLÖCHL, Phys. Rev. B, 50, 17953 (1994).

61. K. A. FICHTHORN and W. H. WEINBERG, J. Chem. Phys., 95, 1090 (1991).

62. A. B. BORTZ, M. H. KALOS, and J. L. LEBOWITZ, J. Comput. Phys., 17, 10 (1975).

63. S. T. PICRAUX and F. L. VOOK, J. Nucl. Mater., 53, 246 (1974).

64. S. T. PICRAUX and F. L. VOOK, Phys. Rev. Lett., 33, 1216 (1974).

65. E. LIGEON, R. DANIELOU, J. FONTENILLE, and R. EYMERY, J. Appl. Phys., 59, 108 (1986).

66. S. NAGATA and K. TAKAHIRO, J. Nucl. Mater., 283287, 1038 (2000).

67. M. ALONSO and E. FINN, Fundamental University Physics Volume III: Quantum and Statistical Physics, AddisonWesley, Reading, Massachusetts (1968).

68. J. T. M. DE HOSSON, A. W. SLEESWYK, L. M. CASPERS, W. VAN HEUGTEN, and A. VAN VEEN, Sol. State Comm., 18, 479 (1976).

69. W. D. WILSON and C. L. BISSON, Radiat. Eff., 19, 53 (1973).

FUSION SCIENCE AND TECHNOLOGY VOL. $50 \quad$ JULY 2006 
70. D. J. REED, Radiat. Eff., 31, 129 (1977).

71. R. A. ANDERL, D. F. HOLLAND, G. R. LONGHURST, and R. J. PAWELKO, Fusion Technol., 21, 745 (1992).

72. F. MAURY, M. BIGET, P. VAJDA, A. LUCASSON, and P. LUCASSON, Radiat. Eff., 38, 53 (1978).
73. M. S. BLANTER, Phys. Status Solidi B, 200, 423 (1997).

74. D. A. KOMAROV, A. V. MARKIN, S. Y. RYBAKOV, and A. P. ZAKHAROV, J. Nucl. Mater., 290-293, 433 (2001).

75. J. R. FRANSENS, M. S. A. E. KERIEM, and F. PLEITER, J. Phys. Condens. Matter, 3, 9871 (1991). 